\title{
Observation on Clinical Efficacy and Safety of Amiodarone in the Treatment of Malignant Arrhythmia after Acute Myocardial Infarction (AMI)
}

\author{
Lu Jiazhong
}

Fuyang City, Anhui Province Second People's Hospital, Fuyang, Anhui 236015

Abstract: To investigate the clinical efficacy and safety of ethylamine iodine in the treatment of malignant arrhythmia after acute myocardial infarction. Method: 35 patients with malignant arrhythmia after acute myocardial infarction were enrolled in our hospital from May 2013 to August 2014. The patients were treated with ethidium iodide load of 75-150 mg/times. The patients were treated with intravenous infusion of $0.5-1.0 \mathrm{mg} / \mathrm{min}$ micro pump at $15-20 \mathrm{~min}$. The clinical curative effect and the heart rate were analyzed at $15 \mathrm{~min}, 1,2$ and $24 \mathrm{~h}$ after treatment respectively, mean arterial pressure changes and adverse reactions and so on. Results: The total effective rate was $91.43 \%$ in the clinical treatment. The heart rate, mean arterial pressure and the difference before treatment were significant $(\mathrm{P}<0.05)$ at $15 \mathrm{~min}$, 1,2 , and $24 \mathrm{~h}$ after treatment and the drug dose was adjusted in 3 patients after bradycardia after return to the normal range. Conclusion: Ethylamine iodine can be used in the clinical treatment of acute myocardial infarction and malignant arrhythmia. It can achieve significant clinical curative effect, high safety and mild adverse reaction. It is worthy to be popularized and applied.

Keywords: ethylamine iodine; acute myocardial infarction; malignant arrhythmia; clinical efficacy

\section{Introduction}

In recent years, the prevalence of acute myocardial infarction gradually increased, after its occurrence often associated with malignant rapid arrhythmia, is a critical clinical and critical disease [1]. At present, the clinical selection of ethylamine iodine as antiarrhythmic treatment drugs, can effectively prevent the phenomenon of potassium outflow, to promote the action potential time limit increases, but also can prevent calcium outflow, in the $\beta$ receptor to play non-competitive Inhibitory effect. In present time, ethylamine iodine is a drug that is clinically effective in the treatment of antiarrhythmic drugs with a negative inotropic effect and is less potent. In this study, 35 patients with acute myocardial infarction with malignant arrhythmia in patients with clinical treatment of ethylamine iodine given to the treatment of its application to be analyzed, the specific content is as follows.

\section{Materials and method}

\subsection{General information}

The study was conducted in 35 patients with malignant arrhythmia after acute myocardial infarction in our hospital from May 2013 to August 2014. Among them, 20 were male and 15 were female and 41-78 were the between the average age $(59.5 \pm 6.2)$. All patients with disease diagnosis and the Chinese Medical Association in 2001 to develop the relevant standards. Heart function NYHA classification: grade 5 patients; II grade 15 patients, III grade 10 patients, IV grade 5 patients; infarct site: 15 cases of anterior wall, 5 cases of inferior wall, 8 cases of anterior wall and lower wall, 3 cases of inferior wall and posterior wall, 4 cases of inferior wall and right ventricle; Arrhythmia type: 10 patients with short-term atrial fibrillation accompanied by frequent ventricular contraction; 20 patients with paroxysmal

This is an Open Access article distributed under the terms of the Creative Commons Attribution-Non Commercial 4.0 International License (http://creativecommons.org/ licenses/by-nc/4.0/), permitting all non-commercial use, distribution, and reproduction in any medium, provided the original work is properly cited. 
ventricular tachycardia; 5 patients with ventricular fibrillation, all patients with arrhythmia seizures showed a more obvious blood pressure reduction, serum potassium was $(3.8 \pm 0.3) \mathrm{mmol} / \mathrm{L}$. All patients voluntarily participated in the study and signed informed consent.

\subsubsection{Exclusion criteria ${ }^{[2]}$}

(1) The presence of iodine, receptor blockers allergy history; (2) Suffering from liver, kidney and other physical organ dysfunction and electrolyte imbalance and other diseases; (3) Suffering from sinus node syndrome; (4) II degree or above the atrioventricular block; (5) Suffering from mental illness and prolonged use of sedative drugs.

\subsection{Method}

All patients who were hospitalized were treated with ethylamine iodine and when the arrhythmia was or after defibrillation, the patient was given intravenous injection of 75-150 mg of ethylamine iodofenone injection (Sanofi Winthrop Industrie, National Pharmaceutical Serial Number J20070056), after 15-20min after adjusting the dose to $0.5-1.0 \mathrm{mg} / \mathrm{min}$ speed continued continuous injection therapy. For arrhythmia still no change, you can every $30 \mathrm{~min}$ and then given patients with ethidium iodide baclitaxel $75-150 \mathrm{mg}$ intravenous injection, if sustained within $24 \mathrm{~h}$ no arrhythmia deterioration phenomenon, that can be discontinued observation. Eight patients were treated with dobutamine, dopamine and other drugs due to heart failure, and the dose of ethylamine iodine was not changed. In the course of the whole medication, all patients were given multi-functional ECG monitor on blood pressure, heart rate and other indicators of continuous monitoring, while $15 \mathrm{~min}$ after treatment, 1,2,24 $\mathrm{h}$ heart rate, mean arterial pressure changes and adverse reactions situation to observe.

\subsection{Observe indicators}

The clinical curative effect was observed. The changes of heart rate, mean arterial pressure and curative effect and adverse reaction were observed at $15 \mathrm{~min}, 1,2$ and $24 \mathrm{~h}$ after treatment.

Disease clinical efficacy criteria ${ }^{[3]}$ : (1) Remarkably effective: The clinical symptoms were significantly improved, ventricular arrhythmia disappeared, or ventricular premature beats and the frequency of treatment before the reduction of more than $90 \%$, arrhythmia converted to sinus; (2) Effective: The patient's clinical symptoms have been reduced, significantly slower heart rate. The incidence of ventricular arrhythmia was lower than that of the previous day, and the number of ventricular premature beats was lower than that of the control group $(\mathrm{P}<0.05)$. (3) Invalid: The patient had no change in the condition, the time of arrhythmia was changed, gradually increased. The total effective rate is (significant number of cases + valid cases) / total number of cases $* 100 \%$.

\subsection{Statistical analysis}

All the data in the study were analyzed by SPSS18.0 statistical software. The count data were expressed as "n (\%)". The test was performed by $\mathrm{x} 2$ test. The data were expressed as "mean \pm standard deviation", and the $\mathrm{t}$ test method validation. If $\mathrm{P}<0.05$, it means that there is statistical significance.

\section{Results}

\subsection{To observe the different types of patients with arrhythmia clinical treatment}

All patients were treated with sinus rhythm for ventricular fibrillation $(84.7 \pm 17.5)$ times/min; using amiodarone time $(36.3 \pm 6.5) \mathrm{h}$, the dose was $(213.0 \% 65.1) \mathrm{mg}$ in 5 cases of ventricular fibrillation effective in 5 cases, ineffective in 2 cases; short-term atrial fibrillation and frequent ventricular premature astigmatism markedly effective in 8 cases, effective in 11 cases, ineffective in 1 case, the total effective rate was $91.43 \%$.

\subsection{Observation and treatment after $15 \mathrm{~min}, 1,2,24 \mathrm{~h}$ heart rate and mean arterial pressure changes}

Compared with before treatment, the heart rate and average arterial pressure were improved at $15 \mathrm{~min}, 1,2$ and $24 \mathrm{~h}$ after treatment, and the difference was statistically significant $(\mathrm{P}<0.05)$. 
Table 1 analysis of 15 minutes after treatment, 1,2,24 h heart rate and mean arterial pressure changes

\begin{tabular}{|c|c|c|c|c|c|}
\hline \multirow{2}{*}{ Group } & \multirow{2}{*}{ Before treatment } & \multicolumn{4}{|c|}{ After treatment } \\
\hline & & $15 \mathrm{~min}$ & $1 \mathrm{~h}$ & $2 \mathrm{~h}$ & $24 h$ \\
\hline $\begin{array}{l}\text { Heart rate/ } \\
(\text { times } / \mathrm{min})\end{array}$ & $138.8 \pm 28.4$ & $105.4 \pm 11.8$ & $89.6 \pm 15.8$ & $89.6 \pm 15.8$ & $72.5 \pm 14.0$ \\
\hline $\begin{array}{c}\text { Mean arterial } \\
\text { pressure/ ( mmhg })\end{array}$ & $48.2 \pm 9.6$ & $60.2 \pm 10.2$ & $72.6 \pm 10.8$ & $83.8 \pm 9.0$ & $89.5 \pm 9.6$ \\
\hline
\end{tabular}

\subsection{To observe the occurrence of adverse reactions in patients}

In the course of the whole treatment did not occur QT interval prolongation, cardiac function deterioration, ventricular arrhythmia increased; 3 cases of slower heart rate in patients with reduced dose of heart rate gradually returned to normal range.

\section{Discussion}

Acute myocardial infarction in Europe and the United States is the most common, about 1.5 million per year suffering from myocardial infarction. In recent years, the incidence of the disease in the country gradually increased. The pathogenesis is due to acute coronary artery disease, persistent ischemia, hypoxia caused by myocardial necrosis symptoms, patients often show sternal after severe, persistent pain, accompanied by increased serum myocardial activity, ECG and other symptoms, and can be combined with arrhythmia, heart failure and other complications, a serious threat to the lives of patients ${ }^{[4]}$. In clinical diagnosis, due to the characteristics of occult disease in acute myocardial infarction and do not have specific causes of increased mortality in patients, especially in patients with malignant arrhythmia. Therefore, should be given timely and effective drug treatment, improve clinical efficacy and improve the prognosis.

With the continuous development of science and technology and medical equipment and the level of continuous improvement and improvement, making coronary intervention is widely used, thereby enhancing the success rate of acute cardiac infarction rescue, but with malignant arrhythmia symptoms, especially acute myocardial ventricular fibrillation after infarction and ventricular tachycardia has gradually become the main cause of death ${ }^{[5]}$. There have been relevant studies reported that malignant ventricular arrhythmias generally occur in the myocardial infarction after a few hours, the incidence of $53.64 \%{ }^{[6]}$. After acute myocardial infarction with malignant arrhythmia pathogenesis, including the following: (1) Due to the patient's myocardial cells in the ischemic, necrotic state, resulting in local myocardial electrophysiological changes in the structure, so that the cardiomyocyte membrane $\mathrm{Na}+, \mathrm{K}+$ ion pump dysfunction and trigger activities, and ultimately lead to myocardial cell activity that is difficult to maintain a balance and directly lead to malignant arrhythmia; (2) Myocardial ischemia, necrosis caused by the accumulation of metabolites in vivo, metabolic acid-base balance disorders, catecholamine secretion increased and hypoxemia and other phenomena, and thus indirectly caused by arrhythmia; (3) Patients with autonomic nervous system activity than ever before, myocardial ischemia to promote endogenous catecholamines in the body and the sympathetic nervous system is highly activated, prone to malignant arrhythmia; (4) Patients with acute myocardial infarction its ischemic, hypoxia and cardiac output decreased, left ventricular pressure, increased capacity caused by increased ventricular wall tension, the impact of myocardial electrophysiological characteristics, making ventricular fibrillation threshold decreased and induced malignant ventricular arrhythmias ${ }^{[7]}$.

In the clinical mechanism of malignant arrhythmia, given the type of disease patients with reasonable and scientific antiarrhythmic drugs to improve the prognosis has an important positive effect. Ethylamine iodine is a complex antiarrhythmic drug with all four classes of Vaughn Williams antiarrhythmic drugs. It has a more complex electrophysiological effect and can effectively inhibit the production of sodium channels and calcium channels in patients. APD, BRP extension, while the o-phase rise rate of a significant decline ${ }^{[8]}$. In addition, it can block the sympathetic $\beta$ receptor and $\alpha$ receptor and make the heart rate slow, dilate the characteristics of vascular smooth muscle. Ethylamine iodine has an inhibitory effect on the patients' reflexive agglutination, which can promote the prolongation of effective refractory period, inhibit the autonomy of sinus node and significantly reduce the peripheral blood resistance, increase the coronary blood flow and reduce the patient's myocardial oxygen consumption and reduce ventricular rate, fully to achieve the purpose of treatment of arrhythmia. 
It has been suggested that the use of ethylamine iodine in the treatment of patients with acute myocardial infarction with malignant ventricular arrhythmias can reduce the mortality of arrhythmias in patients with myocardial infarction. Compared with other drugs, amiodarone can help patients achieve the desired clinical efficacy and prognosis ${ }^{[9]}$. The results of this study found that ethylamine iodine treatment of acute myocardial infarction after malignant ventricular arrhythmia clinical effect is more significant, can effectively reduce the risk of cardiac death, ventricular arrhythmias. It can be seen that ethylamine iodine is clinically effective in preventing ventricular tachycardia, ventricular fibrillation occurred in the first wash drug. However, due to the traditional ethylamine iodine oral treatment with slow absorption, long half-life defects and make the drug slow, for patients with malignant tachyarrhythmias in patients with unstable hemodynamics clinical treatment is difficult to achieve the desired results. In addition, it was suggested that administration of intravenous injection of ethylamine iodine injection in patients could reduce the risk of recurrence in patients after cardioversion. In this study, 35 cases of acute myocardial infarction after the malignant arrhythmia to give ethylamine iodine load, prompting the patient's myocardial tissue in the higher plasma concentration can be fully exposed, through the use of micro-pump to effectively maintain the plasma concentration, to promote the plasma concentration is always at the peak and the fastest speed to achieve effective blood concentration, on the other hand to ensure that the plasma concentration of sustained and stable, the maintenance of sinus rhythm have a positive effect. The results showed that the total effective rate of amiodarone was $91.43 \%, 15 \mathrm{~min}$ after treatment, 1, 2, $24 \mathrm{~h}$ heart rate and mean arterial pressure before and after treatment, the difference was significant $(\mathrm{p}<0.05)$, indicating that the way through the micro-pump in patients with arrhythmia after acute myocardial infarction intravenous injection of ethylamine iodine clinical efficacy is good, and no inhibition of cardiac pump function; when the malignant arrhythmia has improved, still continued intravenous $24 \mathrm{~h}$ after injection, can reduce the recurrence rate of arrhythmia.

Studies have suggested that ethylamine iodine caused to low blood pressure and its lysing enzyme in a certain relationship, to promote the slow pace of intravenous injection and reduce hypotension ${ }^{[11]}$. In the course of this study, no symptoms of hypotension were observed in all patients. This may be related to the treatment of micro pumps. In the intravenous use of ethylamine iodine treatment, should follow the principle of individual medication, help to reduce the incidence of adverse reactions. 3 patients in the study of bradycardia occurred in the adjustment of drug dose can be returned to their normal level.

In summary, the use of ethylamine iodine in the treatment of acute myocardial infarction after malignant arrhythmia can be significantly improve the clinical efficacy and the prognosis, it is worth applying.

\section{Reference}

1. Zhao Ding Xue. Amiodarone Intravenous Infusion for Arrhythmia Treatment [J]. Contemporary Medicine, 2013,19 (33): 143-144.

2. Chen Shu. The Efficacy of Ethylamine Iodine in the Treatment of Acute Myocardial Infarction Ventricular Arrhythmia 41 Cases [J]. Chinese Journal of Folk Medicine, 2014,23 (4): 39-39.

3. Li Hong. Ethylamine Iodine Combined with Tanshinone II Sodium Sulfonate Injection in the Treatment of Acute Myocardial Infarction Caused by Malignant Arrhythmia [J]. Modern Drugs and Clinical, 2013,28 (4): 566-568

4. Dai Wen Qin. Observation of Ethylamine Iodine Adjuvant Treatment of Acute Myocardial Infarction Complicated by Arrhythmia Clinical Efficacy and Safety [J] .Jilin Medical, 2013,34 (30): 6289-6289.

5. Dong Gui Hong. Ethylamine Iodine Treatment of Acute Myocardial Infarction with Rapid Arrhythmia and Clinical Efficacy and Safety [J]. Inner Mongolia Medical Journal, 2013,45 (9): 1087-1088.

6. Wu Za. Clinical Observation of the Treatment of Acute Myocardial Infarction Complicated with Malignant Arrhythmia [J]. Chinese Journal of Medical Research, 2016,14 (18): 33-34. [J].

7. Fan Ting Ting, $\mathrm{Xu}$ Bang Long. Acute Myocardial Infarction after the New Atrial Fibrillation and Secondary Malignant Arrhythmia Clinical Relevance Analysis [J]. Anhui Medicine, 2015,19 (3): 515-517.

8. Liu Dong Fang. 1 Case of Acute Myocardial Infarction with Malignant Arrhythmia Interventional Care [J]. Chinese Journal of Clinical Nursing, 2012,4 (4): 366-367.

9. Zhang Wen Bo, Li Jia Yi. Myocardial Infarction Acute Anterior Myocardial Infarction after PCI Application of Ethylamine Iodine to Prevent Malignant Arrhythmia [J]. Chinese Journal of Practical Medicine, 2013,8 (6): 136-137.

10. Mo Ni, Chen Hai Jian, Liang Jin Chun. Ethylamine Iodine Treatment of Acute Myocardial Infarction after the Treatment of Malignant Arrhythmia [J]. Chinese Journal of Practical Diagnosis and Treatment, 2012,26 (3): 290-292.

11. Zhou Yan Fang. Acute Myocardial Infarction Early Merger of Malignant Ventricular Arrhythmias in Clinical Treatment Experience [J]. Chinese Medicine Guide, 2015,13 (13): 126-127. 